\title{
Control Adaptativo por Modelo de Referencia para un Inversor basado en LCL con Síntesis de Controlador Mínima
}

\author{
Ciro Larco Barros \\ ciro.larco@ucuenca.edu.ec \\ Ramon Costa Castelló \\ ramon.costa@upc.udu \\ Josep M. Olm. \\ josep.olm@upc.udu
}

\section{Resumen}

Un Control Adaptativo por Modelo de Referencia (MRAC) con Sintesis de Controlador Minima (MCS), es desarrollado con el objetivo de controlar un Inversor monofásico basado en un Filtro $L C L$ conectado a la Red Eléctrica y adaptar el inductor de la red, que es un parámetro desconocido y que genera incertidumbre, manteniendo las prestaciones y la robustez del sistema. Para ello primero se revisara el algoritmo de MCS y en función de este, se muestran todos los pasos a seguir y el desarrollo de los componentes necesarios para el funcionamiento de MCS en tiempo continuo. Se desarrolla un modelo de simulación promediado y se presentan los resultados obtenidos.

Palabras clave: Control Adaptativo por Modelo de Referencia, Minimal Control Síntesis, Filtro LCL, Invertidor de Potencia DC - AC, Energías Alternativas, Incertidumbre Paramétrica, Observador de Estados.

\section{INTRODUCCIÓN}

Bajo el concepto de Generación Distribuida (Figura 1), cada vez más fuentes de Energía Renovable (eólica, fotovoltáica, mareo motriz, pilas de combustible, etc.) son incorporadas a las redes de distribución eléctrica, teniendo como objetivo, contribuir a la gran demanda de energía mundial, y así ir reduciendo la producción de energía por medios contaminantes, como el petróleo, carbón o gas natural, y de esta forma ayudar en la mitigación del calentamiento global. Innumerables estudios alrededor de estos elementos se han llevado a cabo, siendo en este momento los más utilizados los convertidores estáticos de potencia de medio puente y de puente completo [1], dada su simplicidad y el menor número de elementos. El filtro de potencia más utilizado es el LCL, frente alternativas como el filtro L ó LC, debido a su eficacia para eliminar armónicos con pequeños valores de sus elementos y por tanto reducidos tamaños [2], [3]. Puesto que la función del Convertidor es la de transformar una señal de corriente continua $\mathbf{C C}$ en una bipolar ó alterna $\mathbf{C A}$, toma el nombre de Inversor, en tanto que al conjunto inversor y filtro, se le denomina Inversor basado en LCL. Varias alternativas de control se han presentado para el Inversor basado en LCL, tales como asignación de polos [4], [5], compensación de admitancias [6], active damping [7], filtro Notch [8], control Deadbeat [9], controlador $H_{\infty}$ [10], MRAC [11], entre otros.

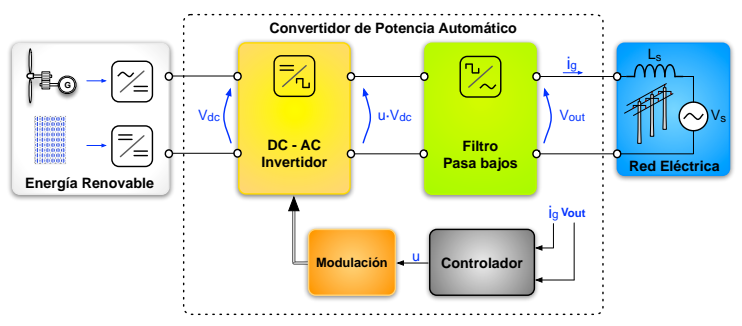

Figura 1: Esquema de Bloques de Generación Distribuida Monofásica

Uno de los inconvenientes presentes para realizar el controlador es que la inductancia de red $L_{s}$ es desconocida; su valor representa el efecto inductivo de la red de baja tensión, por lo que depende de la distancia entre punto de conexión del Inversor y el punto de la Fuente de la Red $V_{s}$, el tipo de sistema, el tipo de configuración de la línea, la distancia entre los conductores, el material conductor y también del diámetro de conductor utilizado para la conexión, así como también de la forma y estructura de la red de distribución. Todo ello hace que el sistema a controlar tenga incertidumbre.

En este trabajo se muestra la implementación de 
un MCS, una variante del control MRAC introducido en [12]. Su principal ventaja sobre el MRAC es la de no necesitar información sobre los valores nominales de los parámetros del sistema, abriendo la posibilidad de controlar plantas que tienen problemas de identificación o incertidumbre en sus parámetros, fallas de modelado y también aquellas que tienen presencia de perturbaciones. Considerando una variación más lenta de los parámetros de la planta con respecto a la dinámica de las ganancias adaptativas, se garantiza un controlador estable y robusto en lazo cerrado [13].

El MCS se ha utilizado en diferentes trabajos prácticos encuadrados en ámbitos de control diversos. En [12] se muestra su aplicabilidad en el control de un motor DC, un depósito de agua y un manipulador clase 1. En [14] se utiliza MCS para el control de la dinámica de vehículos, en [15] se aplica a un sistema de suspensión activa y en [16] se usa para controlar una válvula electrónica de combustible. Se ha usado también MCS para controlar la altitud de satélites [17], y se ha hecho lo propio con el voltaje de salida de un inversor de puente completo en [18].

\section{ALGORITMO DE CONTROL MCS}

Cuando los parámetros de la planta se asumen inciertos y/o con variaciones temporales desconocidas la ley de control dada por el MRAC en [19] debe ajustarse. El algoritmo de MRAC con MCS [12], asume un modelo lineal en el espacio de estados para la planta descrita por:

$$
\dot{\mathbf{x}}(t)=\mathbf{A}_{f c c} \mathbf{x}(t)+\mathbf{B}_{f c c} b u(t)+d(t),
$$

donde $\mathbf{x}(t)$ es el vector de estados del sistema con dimensión $n, u(t)$ es la acción de control con dimensión $m$ y $d(t)$ representa perturbaciones externas desconocidas, no linealidades o términos no modelados del sistema. Por otra parte $\mathbf{A}_{f c c} \mathbf{y} \mathbf{B}_{f c c}$ son matrices de dimensiones apropiadas que deberán estar en forma canónica controlable:

$$
\begin{aligned}
\mathbf{A}_{f c c} & =\left[\begin{array}{cccc}
0 & 1 & \cdots & 0 \\
0 & 0 & \cdots & 0 \\
\vdots & \vdots & \vdots & \vdots \\
0 & 0 & \cdots & 1 \\
-a_{1}(t) & -a_{2}(t) & \cdots & -a_{n}(t)
\end{array}\right] ; \\
\mathbf{B}_{f c c} & =\left[\begin{array}{lllll}
0 & 0 & \cdots & 0 & 1
\end{array}\right] .
\end{aligned}
$$

En esta planta se buscará tener un comportamiento dinámico deseado de acuerdo a las necesidades de trabajo, las cuales se establecen mediante un modelo de referencia lineal que definirá de manera exacta la trayectoria de estado requerida, $\mathbf{x}_{\mathbf{m}}(t)$ :

$$
\dot{\mathbf{x}}_{m}(t)=\mathbf{A}_{m} \mathbf{x}_{m}(t)+\mathbf{B}_{f c c} b_{m} r(t)
$$

donde $r(t)$ es la señal de referencia y tiene la misma dimensión que $u(t)$, en tanto que $\mathbf{x}_{m}$ tiene las mismas dimensiones que $\mathbf{x}$. Además, $\mathbf{A}_{m}$ es una matriz Hurwitz con las mismas dimensiones que $\mathbf{A}_{f c c}$ y en forma canónica de control. Notése que $\mathbf{A}_{m}, b_{m}$ y $r(t)$ vienen establecidos por el diseñador.

La ley de control para MCS viene dada por:

$$
u(t)=\Delta \mathbf{K}(t) \mathbf{x}(t)+\Delta \mathbf{K}_{\mathbf{R}}(t) r(t) .
$$

A partir de (1) y (2) podemos determinar la dinámica del error $\mathbf{x}_{e}(t)$ :

$$
\dot{\mathbf{x}}_{e}(t)=\mathbf{A}_{\mathbf{m}} \mathbf{x}_{e}(t)-d(t)-w_{e}(t)
$$

siendo:

$$
\begin{aligned}
& \mathbf{x}_{e}(t) \triangleq \mathbf{x}_{m}(t)-\mathbf{x}(t) \\
& w_{e}(t)=\mathbf{B}_{f c c}\left[\Delta \mathbf{K}(t) \mathbf{x}(t)+\Delta \mathbf{K}_{\mathbf{R}}(t) r(t)\right]
\end{aligned}
$$

Por último, se establece como salida de la señal de error:

$$
y_{e}(t)=\mathbf{C}_{e}^{T} \mathbf{x}_{e}(t), \quad \mathbf{C}_{e}^{T}=\mathbf{P B}_{f c c},
$$

donde $\mathbf{P}=\mathbf{P}^{T}>0$ es la solución de la ecuación de Lyapunov

$$
\mathbf{P} \mathbf{A}_{m}+\mathbf{A}_{m}{ }^{T} \mathbf{P}=-\mathbf{Q}
$$

con $\mathbf{Q}>0$. Esto garantiza que la terna $\mathbf{A}_{m}, \mathbf{B}_{f c c}, \mathbf{C}_{\mathbf{e}}$ es estrictamente positiva real [20], concepto equivalente al de hiperestabilidad [21] usado originalmente en [19].

Entonces, el error $\mathbf{x}_{e}(t)$ tiende asintóticamente a 0 si se verifica a su vez la desigualdad de Popov:

$$
\int_{t_{0}}^{t_{1}} y_{e}^{T}(t) w_{e}(t) d t \geq-\gamma^{2}, \quad \forall t_{1} \geq t_{0} .
$$

La solución planteada en [19] para satisfacer esta desigualdad es:

$$
\begin{aligned}
\Delta \mathbf{K}(t) & =\int_{0}^{t} \alpha y_{e}(\tau) \mathbf{x}^{T}(\tau) d \tau+\beta y_{e}(t) \mathbf{x}^{T}(t) \\
\Delta \mathbf{K}_{\mathbf{R}}(t) & =\int_{0}^{t} \alpha y_{e}(\tau) r^{T}(\tau) d \tau+\beta y_{e}(t) r^{T}(t)
\end{aligned}
$$


donde $\alpha>0, \beta \geq 0$ son las ganancias de adaptación. Debe, finalmente, tenerse en cuenta que para un perfecto seguimiento del modelo de referencia es necesario que se cumplan las condiciones de Erzberger:

$$
\begin{aligned}
\left(\mathbb{I}_{n}-\mathbf{B}_{m} \mathbf{B}_{f c c}^{\dagger}\right)\left(\mathbf{A}_{\mathbf{f c c}}-\mathbf{A}_{m}\right) & =\mathbf{0}_{n} \\
\left(\mathbb{I}_{n}-\mathbf{B}_{m} \mathbf{B}_{f c c}^{\dagger}\right) \mathbf{B}_{m} & =\mathbf{0}_{n, m},
\end{aligned}
$$

siendo $\mathbb{I}_{n}$ la matriz identidad de dimensión $n \times n$. Así, si los parámetros de la planta varian más lentamente que las ganancias adaptativas se tendrá que el error $\mathbf{x}_{e}$ tiende a 0 de forma globalmente uniformemente asintóticamente estable [13].

\section{IMPLEMENTACIÓN DE MCS PARA EL INVERSOR BASADO EN LCL}

En esta sección se describen cada unos de los elementos necesarios para la implementación de MCS para el Inversor basado en LCL, como también la simulación de los mismos y los resultados obtenidos.

\subsection{FORMA CANÓNICA CONTROLABLE DEL INVERSOR BASADO EN LCL}

Uno de los requerimientos de MCS es representar el sistema a controlar en el espacio de estados en Forma Canónica Controlable, por lo que a partir del diagrama simplificado del Inversor basado en LCL, mostrado en la Figura 2, se procederá a obtener esta estructura.

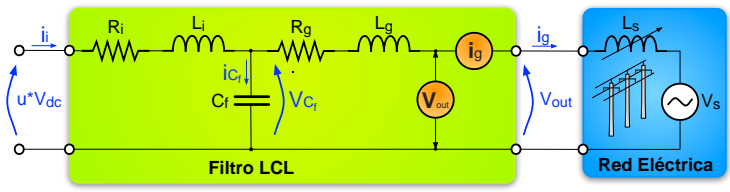

Figura 2: Diagrama simplificado del Inversor basado en LCL

Puesto que el objetivo es controlar la corriente de salida del inversor $i_{g}(t)$, se buscará obtener una expresión que contenga la corriente de salida $i_{g}$ en función del voltaje de entrada $u \cdot V_{d c}$ y el voltaje de Red $V_{s}$. Bajo la consideración de un modelo promediado y mediante la utilización de las leyes de Kirchhoff, se obtienen las siguientes relaciones:

$$
\begin{aligned}
u \cdot V_{d c} & =R_{i} i_{i}+L_{i} \frac{d i_{i}}{d t}+V_{C_{f}} \\
V_{C_{f}} & =R_{g} i_{g}+\left(L_{g}+L_{s}\right) \frac{d i_{g}}{d t}+V_{s} \\
i_{i} & =i_{C_{f}}+i_{g}=C_{f} \frac{d V_{C_{f}}}{d t}+i_{g} .
\end{aligned}
$$

Se definen como variables de estado:

$$
\begin{aligned}
& x_{1} \triangleq i_{g}(t) \\
& x_{2} \triangleq \frac{d i_{g}(t)}{d t}=\frac{d x_{1}}{d t}=\dot{x}_{1} \\
& x_{3} \triangleq \frac{d^{2} i_{g}(t)}{d t}=\frac{d x_{2}}{d t}=\dot{x}_{2} .
\end{aligned}
$$

$\mathrm{Al}$ combinar de manera adecuada las relaciones de (8) y considerando los estados elegidos en (9) se obtiene la realización en el espacio de estados con perturbación:

$$
\begin{aligned}
& \dot{\mathbf{x}}=\mathbf{A} \mathbf{x}+\mathbf{B} u+\mathbf{B}_{\mathbf{0}} V_{s}+\mathbf{B}_{\mathbf{1}} \dot{V}_{s}+\mathbf{B}_{\mathbf{2}} \ddot{V}_{s} \\
& y=\mathbf{C x},
\end{aligned}
$$

que al expandir con sus términos tenemos:

$$
\begin{aligned}
{\left[\begin{array}{c}
\dot{x}_{1} \\
\dot{x}_{2} \\
\dot{x}_{3}
\end{array}\right]=} & {\left[\begin{array}{ccc}
0 & 1 & 0 \\
0 & 0 & 1 \\
-\frac{a_{0}}{a_{3}} & -\frac{a_{1}}{a_{3}} & -\frac{a_{2}}{a_{3}}
\end{array}\right]\left[\begin{array}{c}
x_{1} \\
x_{2} \\
x_{3}
\end{array}\right]+\left[\begin{array}{c}
0 \\
0 \\
\frac{V_{d c}}{a_{3}}
\end{array}\right] u } \\
& +\left[\begin{array}{c}
0 \\
0 \\
\frac{b_{0}}{a_{3}}
\end{array}\right] V_{s}+\left[\begin{array}{c}
0 \\
0 \\
\frac{b_{1}}{a_{3}}
\end{array}\right] \dot{V}_{s}+\left[\begin{array}{c}
0 \\
0 \\
\frac{b_{2}}{a_{3}}
\end{array}\right] \ddot{V}_{s} \\
y= & {\left[\begin{array}{lll}
1 & 0 & 0
\end{array}\right]\left[\begin{array}{c}
x_{1} \\
x_{2} \\
x_{3}
\end{array}\right] }
\end{aligned}
$$

donde se han establecido algunas variables:

$$
\begin{aligned}
& a_{3}=L_{i} C_{f}\left(L_{g}+L_{s}\right) \\
& a_{2}=R_{i} C_{f}\left(L_{g}+L_{s}\right)+L_{i} C_{f} R_{g} \\
& a_{1}=R_{i} C_{f} R_{g}+L_{i}+L_{g}+L_{s} \\
& a_{0}=R_{i}+R_{g} \\
& b_{0}=-1 ; \quad b_{1}=-R_{i} C_{f} ; \quad b_{2}=-L_{i} C_{f} .
\end{aligned}
$$

siendo (10) la representación buscada en el Espacio de Estados en Forma Canónica Controlada para el Inversor basado en LCL. 


\subsection{MODELO DE REFERENCIA PARA EL INVERSOR BASADO EN LCL}

Uno de los elementos más importantes dentro de MCS es el Modelo de Referencia, puesto que este determinará la dinámica del Sistema a controlar, permitiendo al inversor generar una señal de salida con el menor error si los estados entregados por el modelo de referencia son más fieles con respecto a los estados deseados.

El modelo de referencia para el inversor basado en LCL en el espacio de estados vendrá dado por:

$$
\dot{\mathbf{x}}_{m r}(t)=\mathbf{A}_{m r} \mathbf{x}_{m r}(t)+b_{m r} \mathbf{B}_{m r} r(t),
$$

donde la estructura de $\mathbf{A}_{m r}$ y $\mathbf{B}_{m r}$ es:

$$
\mathbf{A}_{m r}=\left[\begin{array}{ccc}
0 & 1 & 0 \\
0 & 0 & 1 \\
-a_{1}^{m} & -a_{2}^{m} & -a_{3}^{m}
\end{array}\right] ; \mathbf{B}_{m r}=\left[\begin{array}{l}
0 \\
0 \\
1
\end{array}\right]
$$

y el vector de estados es el mismo del Inversor:

$$
\mathbf{x}_{m r}(t)=\left[\begin{array}{l}
\dot{i}_{g_{m r}} \\
\dot{i}_{g_{m r}} \\
\ddot{i}_{g_{m r}}
\end{array}\right]=\left[\begin{array}{l}
x_{1 m r} \\
x_{2 m r} \\
x_{3 m r}
\end{array}\right]
$$

El objetivo del Inversor basado en LCL es entregar a la red eléctrica una onda de corriente sinusoidal, lo que implica que el estado $x_{1 m r}$ sea $I_{g_{r e f}}$ sen $w t$, donde $I_{g_{r e f}}$ será la amplitud escogida como salida del Inversor LCL, permitiendo al inversor entregar mas o menos corriente a la Red, en función de la energía generada. Por lo tanto se desea que:

$$
\mathbf{x}_{m r}=\left[\begin{array}{c}
I_{g_{r e f}} \operatorname{sen} w t \\
w I_{g_{r e f}} \cos w t \\
-w^{2} I_{g_{r e f}} \operatorname{sen} w t
\end{array}\right]
$$

Esto se puede conseguir escogiendo:

$$
\begin{aligned}
& r(t)=I_{g_{r e f}} \cos w t ; \\
& A_{m r}=\left[\begin{array}{ccc}
0 & 1 & 0 \\
0 & 0 & 1 \\
-w^{2} & -\left(w^{2}+\frac{1}{L_{i}{ }^{2} C_{f} w}\right) & -1
\end{array}\right] ; \\
& B_{m r}=\frac{1}{L_{i}{ }^{2} C_{f}}\left[\begin{array}{ccc}
0 & 0 & 1
\end{array}\right]^{T} ;
\end{aligned}
$$

y fijando como condiciones iniciales:

$$
C I_{m r}=\left[\begin{array}{llll}
0 & w & I_{g_{r e f}} & 0
\end{array}\right]^{T} .
$$

El modelo referencia obtenido en (12) es controlable, como también observable y la parte real de los valores característicos de $A_{m r}$ se encuentran ubicados todos en el lado izquierdo del plano complejo, garantizando la estabilidad del modelo de referencia. En la Figura 3, se observan los estados generados por el modelo de referencia para el Inversor basado en LCL, los cuales cumplen con los requerimientos de la dinámica deseada, tanto en amplitud como en fase.
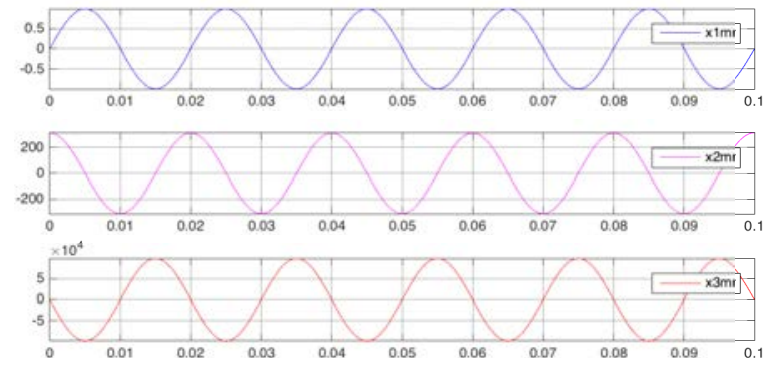

Figura 3: Estados generados por el Modelo de Referencia

\subsection{NORMALIZACIÓN DEL INVERSOR BASADO EN LCL}

Dado que los elementos que conforman el filtro LCL tienen valores muy pequeños y por otro lado, el vector de estados presenta amplitudes muy grandes, es aconsejable normalizar el sistema para así evitar problemas numéricos y a la vez facilitar la búsqueda de valores de $\alpha, \beta$ y $\mathbf{C}_{e}$, dentro de los rangos de implementación.

Para ello se definen las siguientes variables de normalización:

$$
\begin{aligned}
\tau & =\frac{1}{\sqrt{L_{i} C_{f}}} t, & x_{1 N} & =\frac{1}{V_{d c}} \sqrt{\frac{L_{i}}{C_{f}}} i_{g}, \\
x_{2 N} & =\frac{d x_{1 N}}{d \tau}, & x_{3 N} & =\frac{d x_{2 N}}{d \tau} .
\end{aligned}
$$

A partir de (13) se determinan los nuevos estados normalizados del Inversor basado en LCL:

$$
\mathbf{x}_{\mathbf{N}}=\Gamma \mathbf{x},
$$

donde $\boldsymbol{\Gamma}$ es la matriz de normalización:

$$
\boldsymbol{\Gamma}=\frac{1}{V_{d c}} \sqrt{\frac{L_{i}}{C_{f}}}\left[\begin{array}{ccc}
1 & 0 & 0 \\
0 & \sqrt{L_{i} C_{f}} & 0 \\
0 & 0 & L_{i} C_{f}
\end{array}\right]
$$


Para que el sistema pueda variar la amplitud de la corriente de salida del Inversor, se deberá incluir como un factor divisor a $I_{g_{r e f}}$ en la matriz de normalización.

\subsection{OBSERVADOR DE ESTADOS DE ORDEN COMPLETO}

Un aspecto a considerar y superar, es que no todos los estados con los que trabaja el sistema en forma canónica controlable son medibles, por lo que será necesario implementar un observador de estados de orden completo que permita estimar estos estados. Dado que la inductancia de la red $L_{s}$ no es conocida y por otro lado que no es factible medir el valor de voltaje de la red $V_{s}$, se realiza la medición de voltaje en el punto de conexión del Inversor $V_{\text {out }} \mathrm{y}$ la corriente de salida del inversor a controlar $i_{g}$, como se muestra en la Figura 2.

Considerando un modelo promediado y mediante la utilización de las leyes de Kirchhoff, se obtienen las siguientes relaciones:

$$
\begin{aligned}
u \cdot V_{d c} & =R_{i} i_{i}+L_{i} \frac{d i_{i}}{d t}+V_{C_{f}} \\
V_{C_{f}} & =R_{g} i_{g}+L_{i} \frac{d i_{g}}{d t}+V_{\text {out }} \\
i_{i} & =i_{C_{f}}+i_{g}=C_{f} \frac{d V_{C_{f}}}{d t}+i_{g} .
\end{aligned}
$$

Se definen como variables de estado observadas:

$$
\begin{aligned}
& \hat{x}_{1} \triangleq \hat{i}_{g}(t) \\
& \hat{x}_{2} \triangleq \frac{d \hat{i}_{g}(t)}{d t}=\frac{d \hat{x}_{1}}{d t}=\dot{\hat{x}}_{1} \\
& \hat{x}_{3} \triangleq \frac{d^{2} \hat{i}_{g}(t)}{d t}=\frac{d \hat{x}_{2}}{d t}=\dot{\hat{x}}_{2}
\end{aligned}
$$

$\mathrm{Al}$ combinar de manera adecuada las relaciones de (16) y considerando los estados (17) se obtiene la realización en el Espacio de Estados con perturbación para el Observador de Estados:

$$
\begin{aligned}
& \dot{\hat{\mathbf{x}}}=\mathbf{A}_{\mathbf{o}} \hat{\mathbf{x}}+\mathbf{B}_{\mathbf{o}} u+\mathbf{B}_{\mathbf{o}_{0}} V_{\text {out }}+\mathbf{B}_{\mathbf{o}_{1}} \dot{V}_{\text {out }}+\mathbf{B}_{\mathbf{o}_{2}} \ddot{V}_{\text {out }} \\
& y=\mathbf{C} \hat{\mathbf{x}} .
\end{aligned}
$$

que al expandir (18) con sus términos tenemos:

$$
\begin{aligned}
& {\left[\begin{array}{c}
\dot{\hat{x}}_{1} \\
\hat{\hat{x}}_{2} \\
\dot{\hat{x}}_{3}
\end{array}\right]=} {\left[\begin{array}{ccc}
0 & 1 & 0 \\
0 & 0 & \frac{1}{\overline{a_{0}}} \\
-\frac{\overline{a_{1}}}{\overline{a_{3}}} & -\frac{\overline{a_{2}}}{\overline{a_{3}}} & -\frac{\overline{a_{3}}}{\overline{a_{3}}}
\end{array}\right]\left[\begin{array}{l}
\hat{x}_{1} \\
\hat{x}_{2} \\
\hat{x}_{3}
\end{array}\right]+\left[\begin{array}{c}
0 \\
0 \\
\frac{V_{d c}}{\overline{a_{3}}}
\end{array}\right] u } \\
&+\left[\begin{array}{c}
0 \\
0 \\
\frac{b_{0}}{\overline{a_{3}}}
\end{array}\right] V_{\text {out }}+\left[\begin{array}{c}
0 \\
0 \\
\frac{b_{1}}{\overline{a_{3}}}
\end{array}\right] \dot{V}_{\text {out }}+\left[\begin{array}{c}
0 \\
0 \\
\frac{b_{2}}{\overline{a_{3}}}
\end{array}\right] \ddot{V}_{\text {out }} \\
& y=\left[\begin{array}{lll}
1 & 0 & 0
\end{array}\right]\left[\begin{array}{c}
\hat{x}_{1} \\
\hat{x}_{2} \\
\hat{x}_{3}
\end{array}\right]
\end{aligned}
$$

donde se han establecido algunas variables:

$$
\begin{aligned}
& \overline{a_{3}}=L_{i} C_{f} L_{g} \\
& \overline{a_{2}}=R_{i} C_{f} L_{g}+L_{i} C_{f} R_{g} \\
& \overline{a_{1}}=R_{i} C_{f} R_{g}+L_{i}+L_{g} \\
& \overline{a_{0}}=R_{i}+R_{g} \\
& b_{0}=-1 ; \quad b_{1}=-R_{i} C_{f} ; \quad b_{2}=-L_{i} C_{f}
\end{aligned}
$$

y a partir de (18) los estados pueden ser estimados usando el siguiente el Observador:

$$
\begin{aligned}
\dot{\hat{\mathbf{x}}}= & \mathbf{A}_{\mathbf{o}} \hat{\mathbf{x}}+\mathbf{B}_{\mathbf{o}} u+\mathbf{B}_{\mathbf{o}_{\mathbf{0}}} V_{\text {out }}+\mathbf{B}_{\mathbf{o}_{1}} \dot{V}_{\text {out }} \\
& +\mathbf{B}_{\mathbf{o}_{2}} \ddot{V}_{\text {out }}+\mathbf{L}\left(i_{g}-\mathbf{C} \hat{\mathbf{x}}\right),
\end{aligned}
$$

donde $\mathbf{L}$ será el vector de ganancias del Observador. Considerando la inductancia de $\operatorname{Red} L_{s}$ como constante las siguientes relaciones se cumplen:

$$
\begin{aligned}
& V_{\text {out }}=L_{s} \frac{d i_{g}(t)}{d t}+V_{s}=L_{s} \mathbf{H}_{\mathbf{1}} \mathbf{x}+V_{s} \\
& \dot{V}_{\text {out }}=L_{s} \frac{d^{2} i_{g}(t)}{d t}+\dot{V}_{s}=L_{s} \mathbf{H}_{\mathbf{2}} \mathbf{x}+\dot{V}_{s} \\
& \ddot{V}_{\text {out }}=L_{s} \frac{d}{d t}\left(\frac{d^{2} i_{g}(t)}{d t}\right)+\ddot{V}_{s}=L_{s} \mathbf{H}_{\mathbf{2}} \dot{\mathbf{x}}+\ddot{V}_{s} \\
& \ddot{V}_{\text {out }}=L_{s} \mathbf{H}_{\mathbf{2}}\left(\begin{array}{c}
\mathbf{A} \mathbf{x}+\mathbf{B} u+\mathbf{B}_{0} V_{s} \\
+\mathbf{B}_{\mathbf{1}} \dot{V}_{s}+\mathbf{B}_{\mathbf{2}} \ddot{V}_{s}
\end{array}\right)+\ddot{V}_{s},
\end{aligned}
$$

siendo:

$$
\begin{aligned}
& \mathbf{H}_{\mathbf{1}}=\left[\begin{array}{lll}
0 & 1 & 0
\end{array}\right] \\
& \mathbf{H}_{\mathbf{2}}=\left[\begin{array}{lll}
0 & 0 & 1
\end{array}\right] .
\end{aligned}
$$

El vector de error e es definido por:

$$
\mathbf{e}=\mathbf{x}-\hat{\mathbf{x}}
$$

siendo la dinámica del vector de error:

$$
\dot{\mathrm{e}}=\dot{\mathrm{x}}-\dot{\hat{\mathbf{x}}} \text {. }
$$


Al desarrollar (22) se tiene:

$$
\begin{aligned}
\dot{\mathbf{e}}= & \mathbf{A x}+\mathbf{B} u+\mathbf{B}_{\mathbf{0}} V_{s}+\mathbf{B}_{\mathbf{1}} \dot{V}_{s}+\mathbf{B}_{\mathbf{2}} \ddot{V}_{s} \\
& -\mathbf{A}_{\mathbf{o}} \hat{\mathbf{x}}-\mathbf{B}_{\mathbf{o}} u-\mathbf{B}_{\mathbf{o}_{\mathbf{0}}} V_{\text {out }}-\mathbf{B}_{\mathbf{o}_{\mathbf{1}}} \dot{V}_{\text {out }} \\
& -\mathbf{B}_{\mathbf{o}_{\mathbf{2}}} \ddot{V}_{\text {out }}-\mathbf{L}\left(i_{g}-\mathbf{C} \hat{\mathbf{x}}\right)
\end{aligned}
$$

Considerando las siguientes relaciones:

$$
\begin{aligned}
\mathbf{A}_{\mathbf{o}} & =\mathbf{A}-L_{s} \mathbf{B}_{\mathbf{o}_{\mathbf{0}}} \mathbf{H}_{\mathbf{1}}-L_{s} \mathbf{B}_{\mathbf{o}_{\mathbf{1}}} \mathbf{H}_{\mathbf{2}}-\mathbf{B}_{\mathbf{o}_{\mathbf{2}}} \mathbf{H}_{\mathbf{2}} \mathbf{A} \\
\mathbf{B}_{\mathbf{o}} & =\mathbf{B}-L_{s} \mathbf{B}_{\mathbf{o}_{\mathbf{2}}} \mathbf{H}_{\mathbf{2}} \mathbf{B} \\
\mathbf{B}_{\mathbf{o}_{\mathbf{0}}} & =\mathbf{B}_{\mathbf{0}}-L_{s} \mathbf{B}_{\mathbf{o}_{\mathbf{2}}} \mathbf{H}_{\mathbf{2}} \mathbf{B}_{\mathbf{0}} \\
\mathbf{B}_{\mathbf{o}_{1}} & =\mathbf{B}_{\mathbf{1}}-L_{s} \mathbf{B}_{\mathbf{o}_{\mathbf{2}}} \mathbf{H}_{\mathbf{2}} \mathbf{B}_{\mathbf{1}} \\
\mathbf{B}_{\mathbf{o}_{2}} & =\mathbf{B}_{\mathbf{2}}\left(L_{s} \mathbf{H}_{\mathbf{2}} \mathbf{B}_{\mathbf{2}}+\mathbb{I}\right)^{-1}
\end{aligned}
$$

y aplicando las relaciones (21) se obtiene la dinámica del error:

$$
\dot{\mathbf{e}}=\left[\mathbf{A}_{o}-\mathbf{L C}\right] \mathbf{e} .
$$

Por tanto, si $\mathbf{A}_{o}-\mathbf{L C}$ es estable, el vector de error convergerá a cero para cualquier vector de error inicial. En tanto que los polos del observador deberán ser escogidos adecuadamente para que el error de estimación converja lo suficientemente rápido.

\subsection{MODELO DE SIMULACIÓN PROMEDIADO}

El Modelo de Simulación Promediado del Inversor basado en LCL se muestra en la Figura 4, donde se incluye un bloque Fuente de Energía Renovable que tiene una fuente del voltaje controlada que hará las veces del Inversor DC a AC conmutado de Potencia, para así evitar introducir ruidos de conmutación, y a la vez el voltaje $V_{d c}$ a ser controlado, simulará el voltaje de la Fuente de Energía Renovable. El bloque Filtro LCL cuenta con: un Filtro LCL, el sensor de medición del voltaje $V_{\text {out }}$, que es el voltaje en el punto de conexión del Inversor, y el sensor de medición de la corriente de salida $i_{g}$, en el mismo punto. El bloque Red Eléctrica contará con: la inductancia de Red $L_{s}$ que representará y simulará la inductancia presente en el punto de conexión, y la fuente de corriente alterna monofásica $V_{s}$, que representa la Red Eléctrica donde se inyectará la Energía Eléctrica proveniente de la Fuente Energía Renovable. A estos bloques se suman los del Modelo de Referencia, Observador de Estados, Normalización y los bloques de MCS que son la implementación de (3).

Es necesario mencionar que la elección de los valores de $\alpha, \beta$ y $C_{e}$, es una tarea que involucra realizar varias simulaciones hasta que el sistema converja.

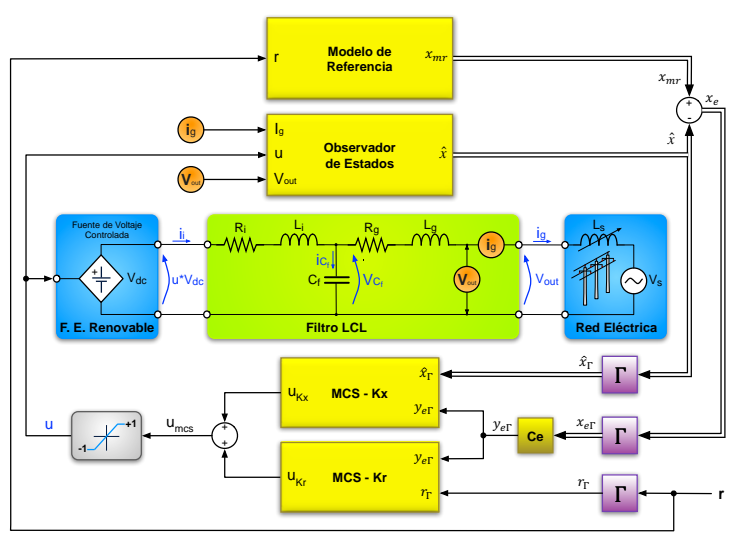

Figura 4: Modelo de Simulación Normalizado Promediado del Inversor basado en LCL con MCS Continuo y Observador de Estados de Orden Completo

Primero se fija una matriz defina positiva $Q$ para obtener $C_{e}$, y luego se varian los valores de $\alpha$ y $\beta$. Si la $Q$ elegida heurísticamente no converge al sistema, se buscará una nueva y se volverá nuevamente a realizar variaciones de $\alpha$ y $\beta$, recomendandose elegir $\alpha=10 \beta$. En tanto que los polos del observador se escogerán suficientemente rápidos, para así no afectar la dinámica del sistema. Las ganancias de adaptación de $\alpha$ y $\beta$, y la matriz definida positiva $Q$ elegidas han sido:

$$
\begin{aligned}
\alpha & =250000, \quad \beta=25000, \\
\mathbf{Q} & =\left[\begin{array}{ccc}
4.94 \cdot 10^{+9} & 0 & 0 \\
0 & 5.46 \cdot 10^{+13} & 0 \\
0 & 0 & 1
\end{array}\right] .
\end{aligned}
$$

Con estos valores de $\mathbf{Q}$ se resuelve la ecuación de Lyapunov (6) y se obtiene $\mathbf{P}$ :

$\mathbf{P}=\left[\begin{array}{lll}2.73 \cdot 10^{+13} & 2.46 \cdot 10^{+09} & 2.50 \cdot 10^{+04} \\ 2.46 \cdot 10^{+09} & 2.73 \cdot 10^{+13} & 2.50 \cdot 10^{+04} \\ 2.50 \cdot 10^{+04} & 2.50 \cdot 10^{+04} & 2.50 \cdot 10^{+04}\end{array}\right]$

que generan los valores:

$$
\mathbf{C}_{e}=\left[\begin{array}{lll}
25026.33 & 25009.21 & 25014.21
\end{array}\right] .
$$

En tanto que los polos del Observador de Estados en lazo cerrado son ubicados en:

$$
s_{1}=-50 W_{r}, \quad s_{2}=-50 W_{r}, \quad s_{3}=-50 W_{r},
$$

siendo $W_{r}$ la frecuencia de resonancia del Filtro LCL $\left(W_{r}=\sqrt{\left(L_{g}+L_{i}\right) / C_{f} L_{g} L_{i}}\right)$. Con esta ubicación de los polos se obtiene las ganancias para el observador:

$$
\mathbf{L}=\left[\begin{array}{lll}
4.05 \cdot 10^{+6} & 5.46 \cdot 10^{+12} & 2.45 \cdot 10^{+18}
\end{array}\right]^{T} .
$$


Utilizando estos valores y considerando una inductancia de la red eléctrica $L_{s}=1 \cdot 10^{-30} \mathrm{H}$, con el objetivo de representar que el Inversor ha sido conectado lo más cerca posible del generador de la Red Eléctrica, se realiza la simulación del esquema de la Figura 4, utilizando el Solver ode23tb (stiff/TRBDF2) con una tolerancia relativa de $1 \cdot 10^{-03}$ y una tolerancia absoluta de $1 \cdot 10^{-06}$ en Simulink, esto por recomendación de SimPowerSystems [22], en tanto que el paso mínimo de integración escogido es $1 \cdot 10^{-10}$, obteniéndose los resultados de la Figura 5. Como se puede observar la corriente $x_{1}$ del inversor sigue correctamente al del modelo de referencia $x_{1 m r}$. En tanto que el error entre los estados $x_{1 m r}$ y $x_{1}$ es muy pequeño, menor al $0.01 \%$, presentando un pequeño salto en el arranque de la simulación.
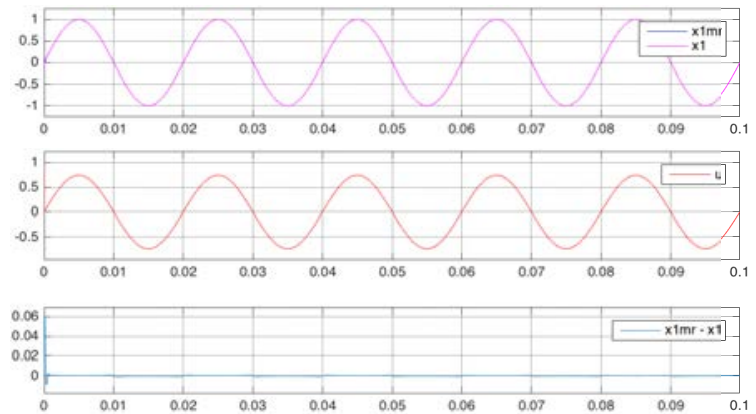

Figura 5: Simulación Modelo Promediado del Inversor basado en LCL con MCS Continuo y Observador de Estados de Orden Completo

En la Figura 6 se presenta la evolución de las ganancias adaptativas $\Delta K_{r}, \Delta K x_{1}, \Delta K x_{2}$ y $\Delta K x_{3}$, mostrando que estás van convergiendo en el tiempo a valores constantes. Al cabo de 10 segundos los valores de las ganancias adaptativas obtenidos de la simulación son: $\Delta K_{r}=0.62, \Delta K x_{1}=42.38$, $\Delta K x_{2}=0.11$ y $\Delta K x_{3}=-0.02$.

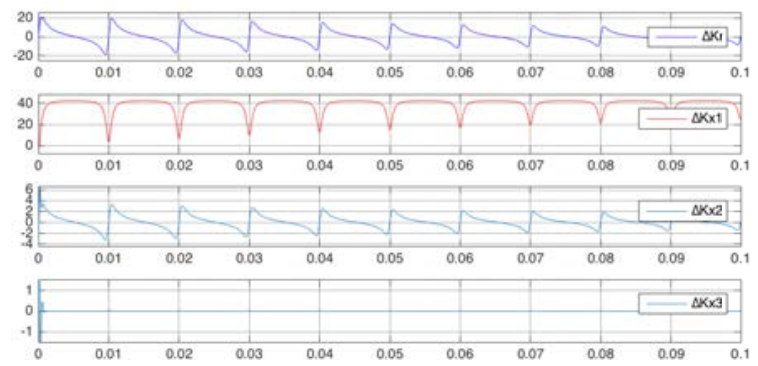

Figura 6: Evolución de las Ganancias Adaptativas $\Delta K_{r}, \Delta K x_{1}, \Delta K x_{2}$ y $\Delta K x_{3}$

Para las simulaciones los elementos del Filtro LCL utilizados han sido: $R_{i}=0.430$ ohmios, $L_{i}=540$ $\mathrm{uH}, R_{g}=0.015 \mathrm{ohmios}, L_{g}=184 \mathrm{uH}, C_{f}=10 \mathrm{uF}$, en tanto que $V_{d c}=420 \mathrm{~V}$ y el voltaje de red $V_{s}=$ $220 \mathrm{~V}$ a una frecuencia de $50 \mathrm{~Hz}$.

\section{Conclusiones}

En este trabajo se ha desarrollado la implementación de un Control Adaptativo por Modelo de Referencia para el control de un Inversor basado en LCL con Síntesis de Controlador Mínima, el cual presenta incertidumbre en el valor del Inductor de red. Numéricamente los resultados muestran que el algoritmo de control funciona correctamente, realizando una rápida adaptación del parámetro desconocido, un seguimiento preciso de la corriente inversor a la del modelo de referencia y un rechazo prefecto de la perturbación del sistema. Se observa también que el error inicial depende de los valores de $\alpha$ y $\beta$, por lo que se los deberá ir incrementando hasta tener un error aceptable. Un mejor arranque y seguimiento se produce cuando los valores de $\mathbf{C}_{e}$ son similares o iguales, un detalle a considerar al momento de elegir los valores de $\mathbf{Q}$.

Esta implementación MCS con un observador de estados abre la posibilidad de desarrollar futuros trabajos en plantas donde la medición de sus estados no es factible.

\section{Agradecimientos}

Este trabajo ha sido realizado gracias al apoyo de la Secretaría Nacional de Educación Superior, Ciencia y Tecnología e Innovación del Ecuador (SENESCYT), a la Universidad de Cuenca, y a los proyectos DPI2013-41224-P y DPI2015- 69286C3-2-R (MINECO/FEDER) y 2014 SGR 267 de la agencia AGAUR de la Generalitat de Catalunya.

\section{Referencias}

[1] N. Mohan, T. M. Undeland, and R. W. P, Power Electronics: Converters, Applications, and Design, vol. 1. México: MC GRAWHILL, third edition ed., Mar 2009.

[2] H. Cha and T. K. Vu, "Comparative analysis of low-pass output filter for single-phase gridconnected photovoltaic inverter," in Applied Power Electronics Conference and Exposition, Twenty-Fifth Annual IEEE, pp. 16591665, Feb 2010.

[3] F. Liu, X. Zha, Y. Zhou, and S. Duan, "Design and research on parameter of LCL filter in three-phase grid-connected inverter," in 
Power Electronics and Motion Control Conference. IEEE 6th International, pp. 21742177, May 2009.

[4] B. Li, M. Zhang, L. Huang, L. Hang, and L. M. Tolbert, "A new optimized pole placement strategy of grid-connected inverter with LCL-filter based on state variable feedback and state observer," in Applied Power Electronics Conference and Exposition, TwentyEighth Annual IEEE, pp. 2900-2906, March 2013.

[5] J. Xu and S. Xie, "Current control based on zero-placement strategy for grid-connected LCL-filtered inverters," in IEEE ECCE Asia Downunder, pp. 1157-1162, June 2013.

[6] C.-L. Chen, J.-S. Lai, Y.-B. Wang, S.-Y. Park, and H. Miwa, "Design and Control for LCL-Based Inverters with Both Grid-Tie and Standalone Parallel Operations," in IAS '08 IEEE Industry Applications Society Annual Meeting, pp. 1-7, Oct 2008.

[7] L. A. Serpa, S. Ponnaluri, P. M. Barbosa, and J. W. Kolar, "A Modified Direct Power Control Strategy Allowing the Connection of Three-Phase Inverters to the Grid Through LCL Filters," IEEE Transactions on Industry Applications, vol. 43, pp. 1388-1400, Sept 2007.

[8] R. Petrella, A. Revelant, and P. Stocco, "A novel proposal to increase the power factor of photovoltaic grid-connected converters at light loads," in Proceedings of the 44th International Universities Power Engineering Conference, pp. 1-5, Sept 2009.

[9] R. Kadri, J.-P. Gaubert, G. Champenois, and M. Mostefai, "Design of a single-phase grid-connected photovoltaic system based on deadbeat current control with LCL filter," in 14th International Power Electronics and Motion Control Conference, pp. T3-147-T3153, Sept 2010.

[10] L. A. Maccari, J. R. Massing, L. Schuch, C. Rech, H. Pinheiro, V. F. Montagner, and R. C. L. F. Oliveira, "Robust $H_{\infty}$ control for grid connected pwm inverters with lcl filters," in 10th IEEE International Conference on Industry Applications, pp. 1-6, Nov 2012.

[11] J. Massing, M. Stefanello, H. Grundling, and H. Pinheiro, "Adaptive Current Control for
Grid-Connected Converters With LCL Filter," IEEE Transactions on Industrial Electronics, vol. 59, pp. 4681-4693, Dec 2012.

[12] Stoten D. P. and Bechoubane H., "Empirical studies of an MRAC algorithm with minimal controller synthesis," Internat J. Control, vol. Vol. 51 (4), pp. 823-849, 1990.

[13] Stoten D. P. and Bechoubane H., "Robustness of a minimal controller synthesis algorithm," Internat J. Control, vol. Vol. 51 (4), pp. 851-861, 1990.

[14] Catino, B., Santini, S., and di Bernardo, M., "MCS adaptive control of vehicle dynamics: an application of bifurcation techniques to control system design," Proceedings 42nd IEEE Conference on Decision and Control, vol. Vol. 3, pp. 2252-2257, 2003.

[15] Hillis A., Harrison A., and Stoten D. P., "A comparison of two adaptive algorithms for the control of active engine mounts," Journal of Sound and Vibration, vol. Vol. 286 (1-2), pp. 37-54, 2004.

[16] di Bernardo M., di Gaeta A., Montanaro U., Olm J. M., and Santini S., "Experimental validation of the discrete-time MCS adaptive strategy," Control Engineering Practice, vol. Vol. 21 (6), pp. 847-859, 2013.

[17] Arif T.T., "A decentralized adaptive control of flexible satellite," Aerospace Conference, IEEE, pp. 3344-3349, 2007.

[18] A. Salvi, S. Santini, D. Biel, J. Olm, and M. di Bernardo, "Model reference adaptive control of a full-bridge buck inverter with minimal controller synthesis," in IEEE 52nd Annual Conference on Decision and Control, pp. 3469-3474, Dec 2013.

[19] Landau, Y. D., Adaptive Control: The Model Reference Approach. New York: Marcel Dekker, 1979.

[20] Khalil, H., Nonlinear Systems, 3rd Ed. Upper Saddle River, NJ: Prentice Hall, 2002.

[21] Popov, V. M., Hyperstability of Automatic Control Systems. New York: Springer-Verlag, 1973.

[22] G. Sybille and P. Brunelle, User's Guide SimPowerSystems For Use with Simulink. Hydro-Québec, Mathworks, 2014. 